\title{
Genetic Mapping of Chartreuse Bulb Color in Onion
}

\author{
Michael J. Havey \\ U.S. Department of Agriculture, Agricultural Research Service and Department of Horticulture, 1575 \\ Linden Drive, University of Wisconsin, Madison, WI 53706
}

\begin{abstract}
AdDitional INDEX wORDs. marker-aided selection, segregation analysis, single nucleotide polymorphisms, unique vegetable color

Abstract. The most common bulb colors of onion (Allium cepa) are red, yellow, and white; chartreuse is a relatively rare bulb color conditioned by the homozygous recessive genotype at the $G$ locus. In this research, plants with chartreuse bulbs were crossed with inbreds with yellow bulbs to develop segregating families for genetic mapping of the $G$ locus. For all of $17 F_{2}$ families, segregations for yellow vs. chartreuse bulbs fit the expected 3:1 ratio $(P>0.05)$. DNAs were isolated from one $F_{2}$ family and genotyped for single nucleotide polymorphisms (SNPs) to produce a genetic map of the $G$ locus and 380 SNPs, of which 119 SNPs have not been previously mapped. Segregations for yellow vs. chartreuse bulbs placed the $G$ locus at the end of chromosome 7 at 6.7 cM from the nearest SNP (isotig28625_2789). This codominant SNP marker linked to the $G$ locus should be useful for introgression of recessive chartreuse bulb color into diverse onion populations for commercial production of this uniquely colored onion.
\end{abstract}

\begin{abstract}
The color of onion (Allium cepa) bulbs is one of the most important consumer traits, and bulb colors can be white, yellow, red, or chartreuse (El-Shafie and Davis, 1967). White bulbs are conditioned by a dominant allele at the inhibitor $(I)$ locus or the homozygous recessive genotype at the color $(C)$ locus, regardless of genotypes at other color loci. Red bulbs are conditioned by dominant alleles at all of the $C, R$, and $L$ loci (El-Shafie and Davis, 1967). Khar et al. (2008) and Duangiit et al. (2014) described a second locus ( $L 2)$ linked to $L$ at which a dominant allele interacts with the $R$ locus to condition red bulbs. Yellow bulbs have a dominant allele at $C$ and the homozygous recessive genotype at one of the loci conditioning red bulbs. The $G$ locus conditions "golden yellow" bulbs when the plant is $i i C$ - (El-Shafie and Davis, 1967), although it is not clear how this color differs from yellow. The homozygous recessive genotype at $G$ conditions chartreuse (light green) bulb color (Fig. 1) in plants that are $i i C$ regardless of genotypes at the $L$ and $R$ loci (El-Shafie and Davis, 1967). Chartreuse bulbs have been reported in the onion cultivars Australian Brown, Greenella, and Giza \#6 (Green et al., 1997; Jones and Mann, 1963). In this research, families segregating for chartreuse bulb color were developed and the $G$ locus mapped relative to SNPs. Molecular markers linked to the $G$ locus should be useful for introgression of recessively inherited chartreuse bulb color into diverse onion populations.
\end{abstract}

\section{Methods}

Two plants with chartreuse bulbs were identified among progenies from the cross MSU5718A $\times$ MSU8155B, which is the female parent of the hybrid 'Sweet Sandwich' (Goldman et al., 2001). These two chartreuse plants were intercrossed

Received for publication 7 Nov. 2019. Accepted for publication 6 Dec. 2019. Published online 21 January 2020.

I thank Christy Stewart and Franco Parisi for technical assistance.

Names are necessary to report factually on available data; however, the U.S. Department of Agriculture (USDA) neither guarantees nor warrants the standard of the product, and the use of the name by USDA implies no approval of the product to the exclusion of others that may also be suitable.

M.J.H. is the corresponding author. E-mail: michael.havey@usda.gov.

This is an open access article distributed under the CC BY-NC-ND license (https://creativecommons.org/licenses/by-nc-nd/4.0/). [breeding plot (BP) 23873]. The cytoplasm and genotype at the nuclear male-fertility restoration $(M s)$ locus (Jones and Clarke, 1943) of chartreuse plants from BP 23873 were established using pooled DNA (described subsequently) and polymorphisms in the chloroplast accD gene (von Kohn et al., 2013) and nuclear AcPms1 marker (Havey and von Kohn, 2017; Kim et al., 2015), respectively. Single progenies from BP 23873 with chartreuse bulbs and waxy foliage were crossed with inbred B5351 with semiglossy foliage or inbred B9885 with glossy foliage (Damon et al., 2014). Seed was harvested separately from both parents and hybrids identified by yellow bulb color from the chartreuse parent or waxy foliage from B5351 and B9885 parents. Individual hybrid progenies were self-pollinated to produce $F_{2}$ families. Bulb colors of $F_{2}$ progenies were scored in field plots as yellow or chartreuse and goodness-of-fit to the expected 3:1 ratio was tested using chi-square analyses. Plants from one $\mathrm{F}_{2}$ family (BP 24334) were self-pollinated and $\mathrm{F}_{3}$ families were grown in field plots and scored for segregations of yellow vs. chartreuse bulb colors. On the basis of segregations in the $F_{3}$ families, the goodness-of-fit to the expected 1:2:1 ratio for the original $F_{2}$ family was tested using chi-square analyses.

DNA was isolated from $F_{2}$ progenies of family BP 24334 using a DNA purification kit (NucleoSpin Plant II Midi; Macherey-Nagel, Düren, Germany) and concentrations established spectrophotometrically (NanoDrop; Thermo Fisher Scientific, Waltham, MA). Single nucleotide polymorphisms (SNPs) were genotyped using DNAs from $\mathrm{F}_{2}$ plants and the Illumina array described by Havey and Ghavami (2018). Goodness-of-fit to the expected 1:2:1 ratio for SNPs were assessed with chi-square analyses and linkages detected at a minimum linkage of logarithm of odds of 12 using Joinmap version 4 (van Ooijen, 2006). Linkage groups were assigned to chromosomes based on 160 SNPs that segregated in earlier mapping populations (Damon and Havey, 2014; Duangjit et al., 2013; Munaiz and Havey, 2020).

\section{Results and Discussion}

All of 85 progenies from the initial cross of two chartreuse plants had chartreuse bulbs, as expected because this bulb color 


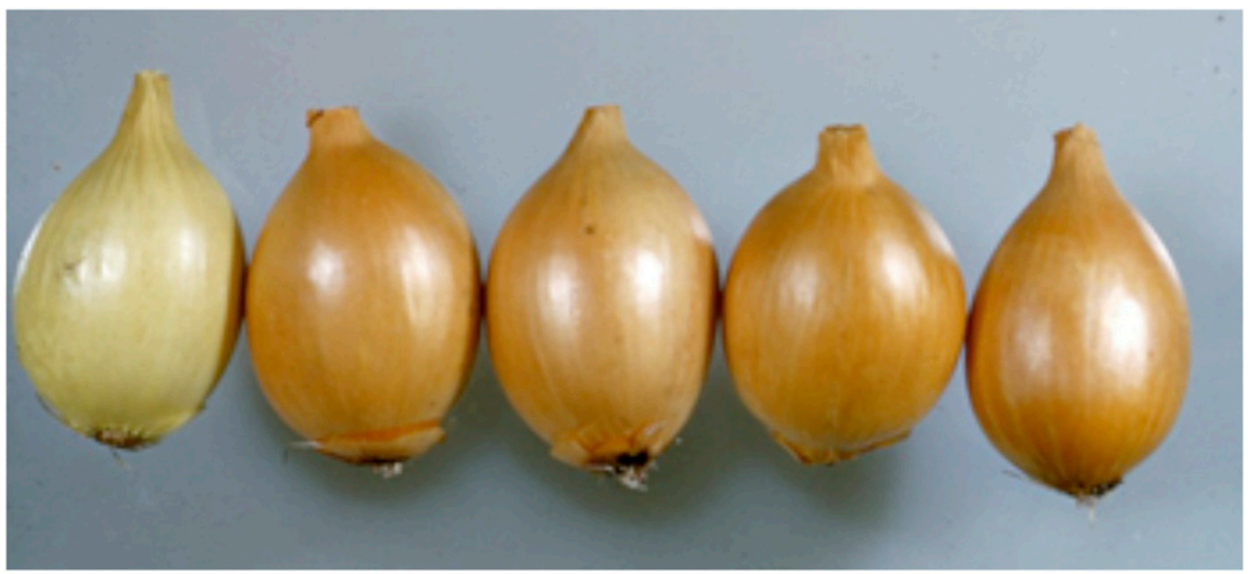

Fig. 1. Chartreuse (left) vs. yellow (right four) bulb colors in onion inbred MSU5718.

Table 1. Observed segregations and probabilities of goodness-of-fit to expected 3:1 ratio for yellow vs. chartreuse (Char) bulbs in $\mathrm{F}_{2}$ families of onion.

\begin{tabular}{cccc}
\hline \multirow{2}{*}{ Cross } & \multicolumn{2}{c}{ Observed } & \\
\cline { 2 - 3 } Yellow & Char & $P$ \\
\hline \multirow{3}{*}{ B5351 $\times$ B5351 Char } & 115 & 26 & 0.072 \\
& 86 & 29 & 0.957 \\
& 34 & 9 & 0.538 \\
& 53 & 21 & 0.502 \\
& 54 & 17 & 0.837 \\
B9885 $\times$ Char & 58 & 29 & 0.073 \\
& 41 & 15 & 0.758 \\
& 48 & 19 & 0.526 \\
& 104 & 34 & 0.922 \\
Char $\times$ B9885 & 107 & 31 & 0.491 \\
& 98 & 22 & 0.092 \\
& 105 & 31 & 0.552 \\
& 85 & 31 & 0.668 \\
& 98 & 23 & 0.128 \\
& 71 & 33 & 0.113 \\
& 101 & 32 & 0.802 \\
& 114 & 43 & 0.489 \\
\hline
\end{tabular}

is conditioned by the homozygous recessive genotype at the $G$ locus (El-Shafie and Davis, 1967). Polymorphisms in chloroplast accD (von Kohn et al., 2013) and nuclear AcPms1 marker (Havey and von Kohn, 2017; Kim et al., 2015) established that the chartreuse plants selected in this study were S cytoplasmic and possessed dominant alleles at the $M s$ locus, indicating that the original chartreuse plants likely originated from self-pollination within cytoplasmic male-sterile line MSU5718A due to the presence of male-fertility restoration allele(s) at the Ms (Jones and Clarke, 1943).

Segregations of yellow vs. chartreuse bulbs fit the expected 3:1 ratio for all of $17 \mathrm{~F}_{2}$ families from crosses of chartreuse plants with inbreds B5351 and B9885 (Table 1), in agreement with the genetic model proposed by El-Shafie and Davis (1967). For $\mathrm{F}_{3}$ families from the cross of a chartreuse plant with B5351, 22 families had only chartreuse bulbs, 46 segregated for chartreuse and yellow bulbs, and 15 families had only yellow bulbs which fit the expected 1:2:1 segregation $[P=0.340$ (Table 2)]. A total of 1624 SNPs (Havey and Ghavami, 2018) were genotyped using DNAs from $92 \mathrm{~F}_{2}$ progenies, of which 380 segregated and all fit the expected 1:2:1 ratio at $P>0.01$ (Table 2). The map locations of 119 previously unmapped SNPs were established (indicated with bold text in Table 2). It was surprising that segregations for bulb colors (Table 1) and all SNPs (Table 2) fit the expected segregation ratios, which may have resulted in parents well adapted to growing conditions in Wisconsin and the robustness of SNP genotyping using the Illumina platform and DNAs from individual plants.

The $G$ locus mapped to the end of chromosome 7 at $6.7 \mathrm{cM}$ distal from SNP isotig28625_2789 (Table 2). Other major loci conditioning bulb colors in onion have been mapped; the $C$ locus maps to chromosome $6, L$ and $L 2$ to chromosome 4 , and $G$ and $R$ to chromosome 7 (Khar et al., 2008; Masuzaki et al., 2006) (Table 2). The $I$ locus showed linkage at $21 \mathrm{cM}$ with ACM006 (Khar et al., 2008), a simple sequence repeat which has not been placed on the genetic map of onion. Khar et al. (2008) reported that the candidate gene for the $R$ locus [dihydroflavonol 4-reductase (Kim et al., 2004)] mapped 11.1 $\mathrm{cM}$ from a restriction fragment length polymorphism revealed by clone AOB212 (King et al., 1998) on chromosome 7. In the genetic map developed by Duangjit et al. (2013), AOB212 mapped $11.2 \mathrm{cM}$ from SNP isotig37252_318 and $27.4 \mathrm{cM}$ from isotig25801_1760. Both of these SNPs segregated in the chartreuse $\times$ B5351 map (Table 2), and comparison of these marker positions and orientations indicate that the $G$ locus should map $\approx 12 \mathrm{cM}$ from $R$ on chromosome 7 .

The colors of vegetables impact consumer acceptance, and consumers may respond positively to uniquely colored vegetables as long as other sensorial characteristics (e.g., flavor, texture, aroma) are acceptable (Leksrisompong et al., 2012). Chartreuse onions rarely appear in markets and may be attractive to consumers interested in uniquely colored vegetables. The SNP marker linked with the $G$ locus on chromosome 7 should be useful for backcrossing recessively inherited chartreuse bulb color into elite onion populations, avoiding the need to self-pollinate after each backcross generation to identify heterozygotes and expediting commercial production of onions with this unique bulb color. 
Table 2. Chromosome (Chrom), position in centiMorgans, observed (Obs) segregations, and probabilities of goodness-of-fit to expected 1:2:1 ratios for single nucleotide polymorphisms (SNPs) and chartreuse vs. yellow bulb colors in an $\mathrm{F}_{2}$ family of onion.

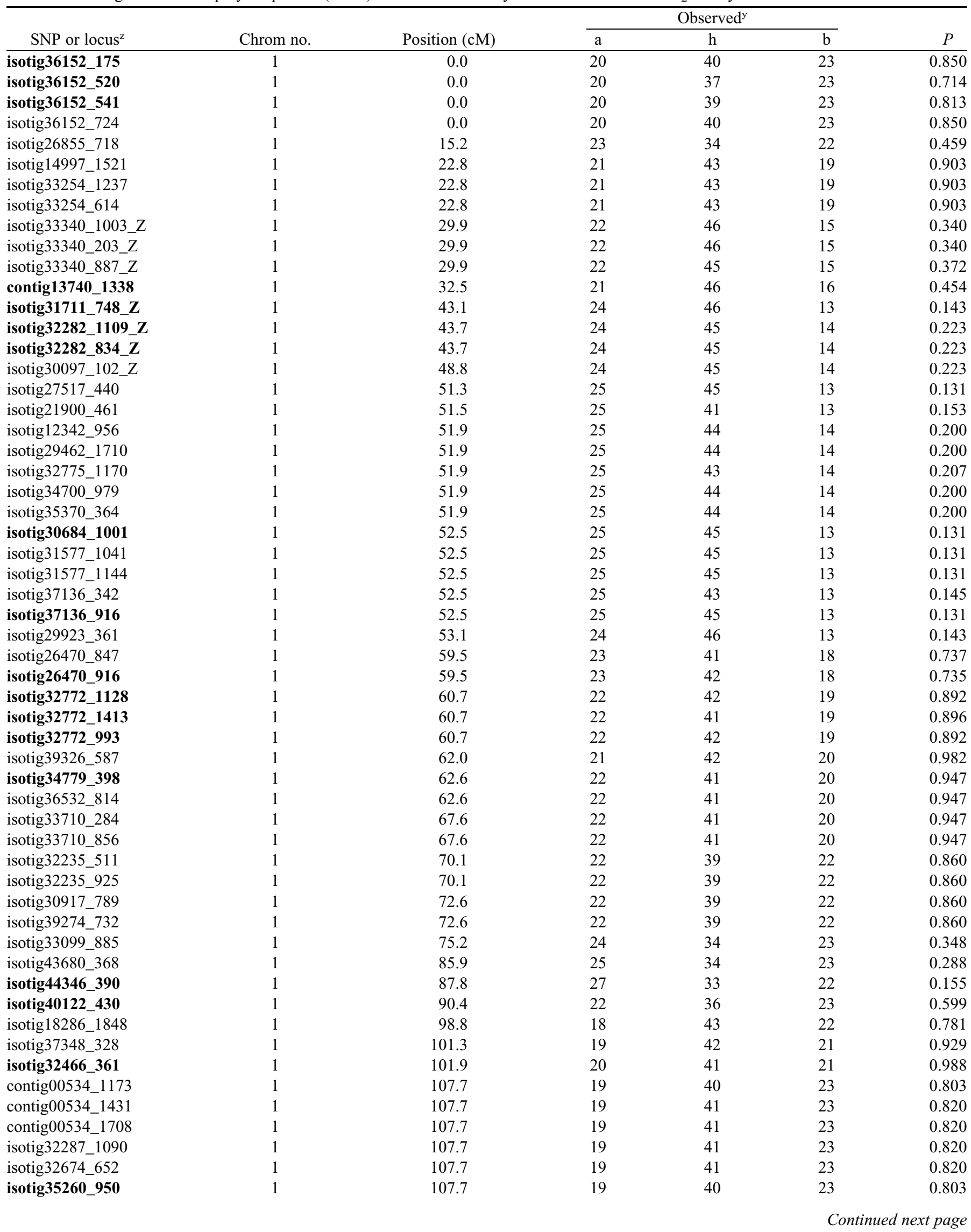


Table 2. Continued.

\begin{tabular}{|c|c|c|c|c|c|c|}
\hline \multirow[b]{2}{*}{ SNP or locus ${ }^{z}$} & \multirow[b]{2}{*}{ Chrom no. } & \multirow[b]{2}{*}{ Position (cM) } & \multicolumn{3}{|c|}{ Observed $^{\mathrm{y}}$} & \multirow[b]{2}{*}{$P$} \\
\hline & & & $\mathrm{a}$ & $\mathrm{h}$ & $\bar{b}$ & \\
\hline isotig38094_540 & 1 & 111.5 & 20 & 39 & 22 & 0.900 \\
\hline isotig37443_625 & 1 & 115.4 & 17 & 44 & 21 & 0.661 \\
\hline isotig32416_877_Z & 2 & 0.0 & 21 & 37 & 23 & 0.703 \\
\hline isotig31747_650_Z & 2 & 11.7 & 21 & 36 & 22 & 0.724 \\
\hline isotig31512_171_Z & 2 & 12.3 & 21 & 41 & 21 & 0.994 \\
\hline isotig22791_480 & 2 & 14.8 & 21 & 39 & 23 & 0.820 \\
\hline contig00172_336 & 2 & 19.2 & 18 & 42 & 23 & 0.735 \\
\hline isotig30979_1313 & 2 & 19.2 & 18 & 42 & 23 & 0.735 \\
\hline isotig30979_525 & 2 & 19.2 & 18 & 42 & 23 & 0.735 \\
\hline isotig35870_429 & 2 & 19.2 & 18 & 42 & 23 & 0.735 \\
\hline isotig35870_603 & 2 & 19.2 & 18 & 42 & 23 & 0.735 \\
\hline contig00087_991 & 2 & 21.0 & 15 & 42 & 23 & 0.407 \\
\hline isotig09466_645 & 2 & 21.0 & 15 & 45 & 23 & 0.344 \\
\hline isotig09466_656 & 2 & 21.0 & 15 & 44 & 23 & 0.368 \\
\hline isotig28648_1787 & 2 & 21.0 & 15 & 45 & 23 & 0.344 \\
\hline isotig28648_2282 & 2 & 21.0 & 15 & 45 & 23 & 0.344 \\
\hline isotig28648_659 & 2 & 21.0 & 15 & 45 & 23 & 0.344 \\
\hline isotig29186_1830 & 2 & 21.0 & 15 & 42 & 23 & 0.407 \\
\hline isotig29645_1947 & 2 & 21.0 & 15 & 45 & 23 & 0.344 \\
\hline isotig30856_1351 & 2 & 21.0 & 15 & 41 & 23 & 0.420 \\
\hline isotig30856_1616 & 2 & 21.0 & 15 & 39 & 23 & 0.433 \\
\hline isotig32987_1114 & 2 & 21.0 & 15 & 42 & 23 & 0.407 \\
\hline isotig32987_979 & 2 & 21.0 & 15 & 41 & 23 & 0.420 \\
\hline isotig33142_579 & 2 & 21.0 & 15 & 45 & 23 & 0.344 \\
\hline isotig37633_597 & 2 & 21.0 & 15 & 45 & 23 & 0.344 \\
\hline isotig17237_4883_Z & 2 & 21.6 & 15 & 42 & 22 & 0.459 \\
\hline isotig19682_515 & 2 & 21.6 & 15 & 46 & 22 & 0.340 \\
\hline isotig25181_1307 & 2 & 21.6 & 15 & 43 & 22 & 0.433 \\
\hline isotig33006_887 & 2 & 21.6 & 15 & 46 & 22 & 0.340 \\
\hline isotig40415_434 & 2 & 25.4 & 16 & 46 & 21 & 0.454 \\
\hline isotig33652_1174 & 2 & 26.0 & 16 & 45 & 22 & 0.482 \\
\hline isotig42036_531 & 2 & 26.7 & 15 & 40 & 22 & 0.499 \\
\hline isotig39285_573 & 2 & 33.3 & 19 & 46 & 18 & 0.607 \\
\hline isotig15060_964 & 2 & 33.9 & 19 & 44 & 19 & 0.803 \\
\hline isotig31233_1053_Z & 2 & 33.9 & 19 & 39 & 19 & 0.994 \\
\hline isotig36775_363_Z & 2 & 35.8 & 18 & 48 & 17 & 0.357 \\
\hline isotig34497_853 & 2 & 42.3 & 24 & 42 & 15 & 0.348 \\
\hline isotig31728_1335 & 2 & 43.0 & 24 & 45 & 14 & 0.223 \\
\hline isotig33627_701 & 3 & 0.0 & 31 & 32 & 20 & 0.026 \\
\hline isotig32949_1359 & 3 & 1.2 & 32 & 32 & 19 & 0.015 \\
\hline isotig32926_115 & 3 & 7.8 & 30 & 34 & 19 & 0.060 \\
\hline isotig34825_1197 & 3 & 9.1 & 29 & 34 & 20 & 0.097 \\
\hline isotig18967_973 & 3 & 12.2 & 31 & 33 & 19 & 0.031 \\
\hline isotig34535_1112 & 3 & 14.0 & 29 & 35 & 18 & 0.095 \\
\hline isotig29415_721 & 3 & 14.1 & 29 & 36 & 18 & 0.112 \\
\hline isotig29570_2011 & 3 & 14.7 & 29 & 35 & 19 & 0.108 \\
\hline isotig31763_1091_Z & 3 & 17.8 & 27 & 38 & 18 & 0.281 \\
\hline isotig31763_1363_Z & 3 & 17.8 & 27 & 38 & 18 & 0.281 \\
\hline isotig31763_1594_Z & 3 & 17.8 & 27 & 36 & 18 & 0.223 \\
\hline isotig31763_874_Z & 3 & 17.8 & 27 & 37 & 18 & 0.252 \\
\hline isotig31763_961_Z & 3 & 17.8 & 27 & 38 & 18 & 0.281 \\
\hline isotig31999_483 & 3 & 17.8 & 27 & 38 & 18 & 0.281 \\
\hline isotig32200_317_Z & 3 & 19.6 & 27 & 39 & 17 & 0.258 \\
\hline isotig32041_963 & 3 & 20.2 & 26 & 40 & 17 & 0.357 \\
\hline isotig33232_1046 & 3 & 20.2 & 26 & 40 & 17 & 0.357 \\
\hline isotig33232_1240 & 3 & 20.2 & 26 & 40 & 17 & 0.357 \\
\hline
\end{tabular}


Table 2. Continued.

\begin{tabular}{|c|c|c|c|c|c|c|}
\hline \multirow[b]{2}{*}{ SNP or locus ${ }^{\mathrm{z}}$} & \multirow[b]{2}{*}{ Chrom no. } & \multirow[b]{2}{*}{ Position (cM) } & \multicolumn{3}{|c|}{ Observed $^{\mathrm{y}}$} & \multirow[b]{2}{*}{$P$} \\
\hline & & & $\mathrm{a}$ & $\mathrm{h}$ & $\mathrm{b}$ & \\
\hline isotig33232_163 & 3 & 20.2 & 26 & 40 & 17 & 0.357 \\
\hline isotig35214_710 & 3 & 20.2 & 26 & 40 & 17 & 0.357 \\
\hline isotig19473_316 & 3 & 20.8 & 26 & 39 & 18 & 0.398 \\
\hline isotig30363_1184 & 3 & 21.4 & 26 & 38 & 19 & 0.412 \\
\hline isotig30363_1478 & 3 & 21.4 & 26 & 38 & 19 & 0.412 \\
\hline isotig30363_1650 & 3 & 21.4 & 26 & 38 & 19 & 0.412 \\
\hline isotig30453_1365 & 3 & 21.4 & 26 & 38 & 19 & 0.412 \\
\hline isotig27896_600 & 3 & 22.0 & 26 & 39 & 18 & 0.398 \\
\hline isotig25849_1264 & 3 & 22.6 & 26 & 40 & 17 & 0.357 \\
\hline isotig25849_1265 & 3 & 22.6 & 26 & 40 & 17 & 0.357 \\
\hline isotig32768_708 & 3 & 22.6 & 26 & 40 & 17 & 0.357 \\
\hline isotig29185_1241 & 3 & 24.5 & 26 & 41 & 16 & 0.298 \\
\hline isotig29185_1803 & 3 & 24.5 & 26 & 41 & 16 & 0.298 \\
\hline isotig29185_2039 & 3 & 24.5 & 26 & 41 & 16 & 0.298 \\
\hline isotig34830_369 & 3 & 26.3 & 28 & 38 & 17 & 0.173 \\
\hline isotig33431_426 & 3 & 27.5 & 29 & 36 & 18 & 0.112 \\
\hline isotig16345_1175 & 3 & 31.9 & 28 & 37 & 18 & 0.184 \\
\hline isotig35180_158 & 3 & 31.9 & 28 & 37 & 18 & 0.184 \\
\hline isotig28422_1511 & 3 & 32.6 & 28 & 36 & 19 & 0.182 \\
\hline isotig28422_191 & 3 & 32.6 & 28 & 36 & 19 & 0.182 \\
\hline isotig34054_350 & 3 & 32.6 & 28 & 36 & 19 & 0.182 \\
\hline isotig31657_1599 & 3 & 39.0 & 25 & 40 & 18 & 0.525 \\
\hline isotig29736_2072_Z & 3 & 42.8 & 26 & 40 & 17 & 0.357 \\
\hline isotig25719_1531_Z & 3 & 44.6 & 23 & 43 & 17 & 0.614 \\
\hline isotig35352_760_Z & 3 & 45.3 & 23 & 42 & 18 & 0.735 \\
\hline isotig35663_382 & 3 & 45.9 & 23 & 41 & 19 & 0.820 \\
\hline isotig35663_432_Z & 3 & 45.9 & 23 & 37 & 18 & 0.655 \\
\hline isotig20004_416 & 3 & 50.9 & 26 & 35 & 22 & 0.298 \\
\hline isotig29785_1760 & 3 & 52.1 & 26 & 37 & 20 & 0.398 \\
\hline isotig30958_160 & 3 & 52.1 & 26 & 37 & 20 & 0.398 \\
\hline isotig33166_330 & 3 & 52.1 & 26 & 37 & 20 & 0.398 \\
\hline isotig34107_268 & 3 & 52.1 & 26 & 37 & 20 & 0.398 \\
\hline isotig34107_828 & 3 & 52.1 & 26 & 37 & 20 & 0.398 \\
\hline isotig35740_1032 & 3 & 52.1 & 24 & 37 & 20 & 0.607 \\
\hline isotig35740_296 & 3 & 52.1 & 26 & 37 & 20 & 0.398 \\
\hline isotig35740_662 & 3 & 52.1 & 26 & 37 & 20 & 0.398 \\
\hline isotig32139_534 & 3 & 55.3 & 25 & 36 & 22 & 0.433 \\
\hline isotig42601_220 & 3 & 55.3 & 25 & 34 & 22 & 0.315 \\
\hline isotig33343_325 & 3 & 61.7 & 23 & 36 & 24 & 0.477 \\
\hline isotig35038_614 & 3 & 62.3 & 22 & 31 & 24 & 0.220 \\
\hline isotig15391_778 & 3 & 73.0 & 23 & 37 & 23 & 0.614 \\
\hline isotig15391_917 & 3 & 73.0 & 23 & 36 & 23 & 0.543 \\
\hline isotig29611_2140 & 3 & 77.4 & 23 & 36 & 24 & 0.477 \\
\hline isotig30944_607 & 3 & 77.4 & 23 & 36 & 24 & 0.477 \\
\hline isotig31416_1679 & 3 & 77.4 & 23 & 36 & 24 & 0.477 \\
\hline isotig35014_773 & 3 & 77.4 & 23 & 34 & 24 & 0.348 \\
\hline isotig33256_667_Z & 3 & 78.0 & 23 & 37 & 23 & 0.614 \\
\hline isotig31068_1490 & 3 & 78.7 & 22 & 36 & 22 & 0.670 \\
\hline isotig32617_1476_Z & 3 & 79.9 & 21 & 38 & 24 & 0.668 \\
\hline isotig33783_1096 & 3 & 87.0 & 23 & 36 & 22 & 0.599 \\
\hline isotig32564_718 & 3 & 87.6 & 22 & 38 & 23 & 0.735 \\
\hline isotig29139_1024 & 3 & 91.4 & 23 & 36 & 24 & 0.477 \\
\hline isotig29139_1381 & 3 & 91.4 & 20 & 40 & 23 & 0.850 \\
\hline isotig29139_1610 & 3 & 91.4 & 20 & 40 & 23 & 0.850 \\
\hline isotig29139_382 & 3 & 91.4 & 20 & 40 & 23 & 0.850 \\
\hline isotig29139_508 & 3 & 91.4 & 20 & 40 & 23 & 0.850 \\
\hline
\end{tabular}


Table 2. Continued.

\begin{tabular}{|c|c|c|c|c|c|c|}
\hline \multirow[b]{2}{*}{ SNP or locus ${ }^{z}$} & \multirow[b]{2}{*}{ Chrom no. } & \multirow[b]{2}{*}{ Position (cM) } & \multicolumn{3}{|c|}{ Observed $^{\mathrm{y}}$} & \multirow[b]{2}{*}{$P$} \\
\hline & & & $\mathrm{a}$ & $\mathrm{h}$ & $\mathrm{b}$ & \\
\hline isotig29139_510 & 3 & 91.4 & 20 & 40 & 23 & 0.850 \\
\hline isotig29680_725 & 3 & 92.0 & 21 & 39 & 23 & 0.820 \\
\hline isotig29251_1141 & 3 & 92.6 & 20 & 36 & 23 & 0.654 \\
\hline isotig29251_1827 & 3 & 92.6 & 20 & 40 & 23 & 0.850 \\
\hline isotig29251_631 & 3 & 92.6 & 20 & 40 & 23 & 0.850 \\
\hline isotig26975_1187 & 3 & 95.1 & 21 & 40 & 22 & 0.936 \\
\hline isotig35099_237_Z & 3 & 96.1 & 19 & 40 & 22 & 0.889 \\
\hline isotig16559_476 & 3 & 98.2 & 19 & 41 & 23 & 0.820 \\
\hline isotig36605_668 & 4 & 0.0 & 20 & 43 & 20 & 0.947 \\
\hline isotig19074_908 & 4 & 0.6 & 20 & 41 & 21 & 0.988 \\
\hline isotig28762_702 & 4 & 3.4 & 20 & 42 & 21 & 0.982 \\
\hline isotig19902_1219 & 4 & 5.4 & 17 & 42 & 21 & 0.741 \\
\hline isotig19902_637 & 4 & 5.4 & 19 & 43 & 21 & 0.903 \\
\hline isotig31547_1040 & 4 & 7.2 & 18 & 44 & 21 & 0.772 \\
\hline isotig31547_1204 & 4 & 7.2 & 18 & 44 & 21 & 0.772 \\
\hline isotig31547_1238 & 4 & 7.2 & 18 & 44 & 21 & 0.772 \\
\hline isotig31547_938 & 4 & 7.2 & 17 & 44 & 21 & 0.661 \\
\hline isotig33497_721_Z & 4 & 11.0 & 16 & 44 & 23 & 0.477 \\
\hline isotig37158_542 & 4 & 14.1 & 15 & 47 & 21 & 0.313 \\
\hline contig00010_596 & 4 & 15.3 & 16 & 44 & 22 & 0.518 \\
\hline isotig28878_757 & 4 & 15.3 & 16 & 45 & 22 & 0.482 \\
\hline isotig30201_786 & 4 & 15.3 & 16 & 44 & 22 & 0.518 \\
\hline isotig26526_748_Z & 4 & 15.9 & 16 & 43 & 23 & 0.499 \\
\hline isotig26526_995_Z & 4 & 15.9 & 16 & 43 & 23 & 0.499 \\
\hline isotig32504_1288 & 4 & 15.9 & 16 & 41 & 23 & 0.529 \\
\hline isotig37005_693 & 4 & 16.2 & 15 & 43 & 23 & 0.389 \\
\hline isotig20995_770 & 4 & 16.5 & 15 & 40 & 23 & 0.429 \\
\hline isotig28741_496 & 4 & 16.5 & 15 & 45 & 22 & 0.372 \\
\hline isotig31736_1067_Z & 4 & 16.5 & 15 & 45 & 23 & 0.344 \\
\hline isotig37005_723 & 4 & 16.5 & 15 & 45 & 23 & 0.344 \\
\hline isotig32123_1465 & 4 & 17.7 & 14 & 45 & 24 & 0.223 \\
\hline isotig37023_265 & 4 & 18.3 & 14 & 46 & 23 & 0.231 \\
\hline isotig33399_1211 & 4 & 18.9 & 14 & 45 & 24 & 0.223 \\
\hline isotig33399_848 & 4 & 18.9 & 14 & 45 & 24 & 0.223 \\
\hline isotig38732_575_Z & 4 & 18.9 & 13 & 45 & 24 & 0.155 \\
\hline isotig37385_830 & 4 & 21.4 & 11 & 49 & 23 & 0.045 \\
\hline isotig44683_192 & 4 & 23.9 & 10 & 47 & 23 & 0.036 \\
\hline isotig44683_264 & 4 & 23.9 & 11 & 49 & 23 & 0.045 \\
\hline isotig31392_1426_Z & 4 & 27.0 & 12 & 47 & 23 & 0.095 \\
\hline isotig35268_1082_Z & 4 & 27.0 & 12 & 48 & 21 & 0.092 \\
\hline isotig35268_784_Z & 4 & 27.0 & 12 & 48 & 23 & 0.084 \\
\hline isotig35268_979_Z & 4 & 27.0 & 12 & 48 & 23 & 0.084 \\
\hline isotig21411_854 & 4 & 27.6 & 12 & 47 & 24 & 0.085 \\
\hline isotig21411_971 & 4 & 27.6 & 12 & 47 & 24 & 0.085 \\
\hline isotig33429_1166 & 4 & 28.8 & 13 & 47 & 23 & 0.145 \\
\hline isotig34748_190 & 4 & 30.7 & 13 & 48 & 22 & 0.136 \\
\hline isotig29269_1573 & 4 & 37.8 & 11 & 51 & 21 & 0.034 \\
\hline isotig36493_410 & 4 & 38.4 & 11 & 51 & 20 & 0.032 \\
\hline isotig31300_1079 & 4 & 39.0 & 12 & 50 & 20 & 0.064 \\
\hline isotig41344_259_Z & 4 & 39.0 & 12 & 50 & 20 & 0.064 \\
\hline isotig41344_432_Z & 4 & 39.0 & 12 & 50 & 20 & 0.064 \\
\hline isotig31846_429 & 4 & 39.6 & 12 & 49 & 21 & 0.078 \\
\hline isotig41409_219 & 4 & 50.4 & 18 & 42 & 23 & 0.735 \\
\hline isotig37821_673 & 4 & 54.2 & 17 & 43 & 22 & 0.669 \\
\hline isotig16136_1083 & 4 & 57.9 & 19 & 44 & 20 & 0.850 \\
\hline isotig16136_752 & 4 & 57.9 & 19 & 44 & 20 & 0.850 \\
\hline
\end{tabular}


Table 2. Continued.

\begin{tabular}{|c|c|c|c|c|c|c|}
\hline \multirow[b]{2}{*}{ SNP or locus ${ }^{z}$} & \multirow[b]{2}{*}{ Chrom no. } & \multirow[b]{2}{*}{ Position (cM) } & \multicolumn{3}{|c|}{ Observed $^{\mathrm{y}}$} & \multirow[b]{2}{*}{$P$} \\
\hline & & & $\mathrm{a}$ & $\mathrm{h}$ & $\mathrm{b}$ & \\
\hline isotig16136_978 & 4 & 57.9 & 19 & 44 & 20 & 0.850 \\
\hline isotig16136_983 & 4 & 57.9 & 19 & 44 & 20 & 0.850 \\
\hline isotig31663_1466 & 4 & 57.9 & 19 & 44 & 20 & 0.850 \\
\hline isotig33886_426 & 4 & 61.8 & 20 & 45 & 14 & 0.295 \\
\hline isotig27765_337 & 4 & 66.3 & 20 & 45 & 18 & 0.709 \\
\hline isotig31106_505 & 4 & 68.8 & 20 & 47 & 16 & 0.398 \\
\hline isotig32888_1192 & 5 & 0.0 & 21 & 46 & 16 & 0.454 \\
\hline isotig26546_969 & 5 & 9.7 & 20 & 44 & 19 & 0.850 \\
\hline isotig28889_2528 & 5 & 17.9 & 20 & 38 & 25 & 0.551 \\
\hline isotig30732_1102 & 5 & 17.9 & 20 & 38 & 25 & 0.551 \\
\hline isotig30732_1647 & 5 & 17.9 & 20 & 37 & 22 & 0.812 \\
\hline isotig33784_560 & 5 & 17.9 & 20 & 38 & 25 & 0.551 \\
\hline isotig17421_2154 & 5 & 23.6 & 17 & 43 & 23 & 0.614 \\
\hline isotig30429_128 & 5 & 23.6 & 17 & 43 & 23 & 0.614 \\
\hline isotig33059_107 & 5 & 25.5 & 17 & 44 & 22 & 0.636 \\
\hline isotig34069_110 & 5 & 26.1 & 16 & 45 & 22 & 0.482 \\
\hline isotig25217_2228 & 5 & 27.3 & 15 & 45 & 23 & 0.344 \\
\hline isotig14741_247_Z & 5 & 27.9 & 15 & 46 & 22 & 0.340 \\
\hline isotig34198_886 & 5 & 29.1 & 14 & 43 & 23 & 0.290 \\
\hline isotig38424_376 & 5 & 29.1 & 14 & 46 & 23 & 0.231 \\
\hline isotig38424_472 & 5 & 29.1 & 14 & 46 & 23 & 0.231 \\
\hline isotig35819_544_Z & 5 & 30.4 & 12 & 48 & 23 & 0.084 \\
\hline isotig37670_180 & 5 & 31.6 & 10 & 50 & 23 & 0.023 \\
\hline isotig32130_277 & 5 & 33.4 & 11 & 49 & 23 & 0.045 \\
\hline isotig35125_448 & 5 & 34.0 & 11 & 46 & 23 & 0.067 \\
\hline isotig30202_736 & 5 & 35.3 & 11 & 50 & 21 & 0.041 \\
\hline isotig30202_796 & 5 & 35.3 & 11 & 50 & 22 & 0.041 \\
\hline isotig30202_919 & 5 & 35.3 & 11 & 50 & 21 & 0.041 \\
\hline isotig32901_1407 & 5 & 35.9 & 11 & 49 & 21 & 0.049 \\
\hline isotig34751_952 & 5 & 35.9 & 11 & 51 & 21 & 0.034 \\
\hline isotig29818_190_Z & 5 & 36.5 & 11 & 45 & 22 & 0.084 \\
\hline isotig29818_808_Z & 5 & 36.5 & 11 & 50 & 22 & 0.041 \\
\hline isotig31385_287_Z & 5 & 37.1 & 10 & 51 & 22 & 0.020 \\
\hline isotig32805_1160 & 5 & 39.6 & 10 & 51 & 22 & 0.020 \\
\hline isotig37875_830 & 5 & 42.7 & 11 & 47 & 22 & 0.065 \\
\hline isotig31152_1676 & 5 & 44.5 & 12 & 50 & 20 & 0.064 \\
\hline isotig38068_242 & 5 & 44.5 & 12 & 51 & 20 & 0.053 \\
\hline isotig06563_373 & 6 & 0.0 & 18 & 46 & 18 & 0.543 \\
\hline isotig31615_250 & 6 & 0.0 & 18 & 47 & 18 & 0.482 \\
\hline isotig34246_1009 & 6 & 0.0 & 18 & 44 & 18 & 0.670 \\
\hline isotig32739_152 & 6 & 1.8 & 20 & 45 & 17 & 0.607 \\
\hline isotig32739_439 & 6 & 1.8 & 20 & 46 & 17 & 0.551 \\
\hline isotig36782_698 & 6 & 1.8 & 18 & 43 & 17 & 0.655 \\
\hline isotig36295_652_Z & 6 & 3.1 & 21 & 44 & 16 & 0.543 \\
\hline isotig28330_2105 & 6 & 7.5 & 20 & 47 & 16 & 0.398 \\
\hline isotig29848_1923 & 6 & 7.5 & 20 & 46 & 16 & 0.447 \\
\hline isotig30657_998 & 6 & 7.5 & 20 & 47 & 16 & 0.398 \\
\hline isotig20052_1261 & 6 & 11.2 & 21 & 45 & 17 & 0.614 \\
\hline isotig31703_857 & 6 & 11.2 & 21 & 45 & 17 & 0.614 \\
\hline isotig32152_614 & 6 & 13.1 & 18 & 47 & 16 & 0.335 \\
\hline isotig31280_1328 & 6 & 23.8 & 21 & 44 & 18 & 0.772 \\
\hline isotig31280_1391 & 6 & 23.8 & 21 & 44 & 18 & 0.772 \\
\hline isotig31280_1610 & 6 & 23.8 & 21 & 44 & 18 & 0.772 \\
\hline isotig35768_1013 & 6 & 25.0 & 21 & 44 & 18 & 0.772 \\
\hline isotig28527_2500 & 6 & 28.8 & 22 & 42 & 17 & 0.695 \\
\hline isotig32066_1149 & 6 & 30.6 & 22 & 43 & 18 & 0.781 \\
\hline
\end{tabular}




\begin{tabular}{|c|c|c|c|c|c|c|}
\hline \multirow[b]{2}{*}{ SNP or locus ${ }^{z}$} & \multirow[b]{2}{*}{ Chrom no. } & \multirow[b]{2}{*}{ Position (cM) } & \multicolumn{3}{|c|}{ Observed $^{\mathrm{y}}$} & \multirow[b]{2}{*}{$P$} \\
\hline & & & $\mathrm{a}$ & $\mathrm{h}$ & $\mathrm{b}$ & \\
\hline isotig32066_1385 & 6 & 30.6 & 22 & 43 & 18 & 0.781 \\
\hline isotig32066_515 & 6 & 30.6 & 22 & 43 & 18 & 0.781 \\
\hline isotig32066_821 & 6 & 30.6 & 22 & 42 & 18 & 0.803 \\
\hline isotig10023_2319 & 6 & 34.4 & 20 & 41 & 20 & 0.994 \\
\hline isotig31550_508_Z & 6 & 40.2 & 22 & 37 & 21 & 0.789 \\
\hline isotig31550_658_Z & 6 & 40.2 & 22 & 39 & 21 & 0.896 \\
\hline isotig31550_670_Z & 6 & 40.2 & 22 & 40 & 21 & 0.936 \\
\hline isotig16577_681 & 6 & 42.4 & 19 & 43 & 21 & 0.903 \\
\hline isotig28276_1535 & 6 & 46.5 & 20 & 42 & 21 & 0.982 \\
\hline isotig28276_320 & 6 & 46.5 & 20 & 42 & 21 & 0.982 \\
\hline isotig30163_1380 & 6 & 47.7 & 19 & 44 & 20 & 0.850 \\
\hline isotig35291_456 & 6 & 50.8 & 16 & 44 & 19 & 0.534 \\
\hline isotig34877_391 & 6 & 60.9 & 17 & 46 & 20 & 0.551 \\
\hline isotig35250_340 & 6 & 62.1 & 16 & 48 & 19 & 0.324 \\
\hline isotig30463_150 & 6 & 64.6 & 15 & 46 & 22 & 0.340 \\
\hline isotig30463_249 & 6 & 64.6 & 15 & 46 & 22 & 0.340 \\
\hline isotig28768_2758 & 6 & 65.8 & 15 & 46 & 22 & 0.340 \\
\hline isotig30686_364 & 6 & 70.9 & 15 & 48 & 20 & 0.267 \\
\hline isotig30686_371 & 6 & 70.9 & 14 & 46 & 20 & 0.259 \\
\hline isotig30686_719 & 6 & 70.9 & 15 & 48 & 20 & 0.267 \\
\hline isotig31045_384_Z & 6 & 70.9 & 15 & 48 & 20 & 0.267 \\
\hline isotig26300_278 & 6 & 78.0 & 17 & 45 & 21 & 0.614 \\
\hline isotig36354_189 & 6 & 78.0 & 17 & 42 & 21 & 0.741 \\
\hline isotig29703_1814 & 6 & 86.5 & 18 & 44 & 21 & 0.772 \\
\hline isotig30292_771 & 6 & 86.5 & 18 & 43 & 21 & 0.813 \\
\hline isotig30464_1503 & 6 & 86.5 & 18 & 44 & 21 & 0.772 \\
\hline isotig30464_1566 & 6 & 86.5 & 18 & 44 & 21 & 0.772 \\
\hline isotig30464_804 & 6 & 86.5 & 18 & 44 & 21 & 0.772 \\
\hline isotig31266_1737 & 6 & 86.5 & 18 & 44 & 21 & 0.772 \\
\hline isotig31266_696 & 6 & 86.5 & 18 & 44 & 21 & 0.772 \\
\hline isotig31266_752 & 6 & 86.5 & 18 & 44 & 21 & 0.772 \\
\hline isotig33111_603 & 6 & 86.5 & 18 & 44 & 21 & 0.772 \\
\hline isotig45729_183 & 6 & 86.5 & 18 & 44 & 21 & 0.772 \\
\hline isotig37324_542 & 6 & 90.3 & 15 & 46 & 18 & 0.306 \\
\hline isotig30426_1109 & 6 & 94.9 & 18 & 47 & 18 & 0.482 \\
\hline isotig38248_370 & 7 & 0.0 & 18 & 45 & 18 & 0.607 \\
\hline isotig35721_667_Z & 7 & 2.6 & 21 & 44 & 18 & 0.772 \\
\hline isotig42233_514_Z & 7 & 12.5 & 18 & 44 & 21 & 0.772 \\
\hline isotig32031_755 & 7 & 18.2 & 18 & 45 & 20 & 0.709 \\
\hline isotig29081_692 & 7 & 25.3 & 19 & 40 & 20 & 0.981 \\
\hline isotig29157_1045 & 7 & 32.4 & 15 & 50 & 18 & 0.157 \\
\hline isotig29157_1114 & 7 & 32.4 & 15 & 50 & 18 & 0.157 \\
\hline isotig29157_1276 & 7 & 32.4 & 15 & 49 & 18 & 0.188 \\
\hline isotig29157_1297 & 7 & 32.4 & 15 & 50 & 18 & 0.157 \\
\hline isotig29157_1381 & 7 & 32.4 & 15 & 50 & 18 & 0.157 \\
\hline isotig36575_674 & 7 & 32.4 & 16 & 49 & 18 & 0.246 \\
\hline isotig36575_914 & 7 & 32.4 & 16 & 46 & 18 & 0.387 \\
\hline isotig42387_377 & 7 & 34.2 & 17 & 47 & 19 & 0.460 \\
\hline isotig34668_1112_Z & 7 & 35.5 & 19 & 45 & 19 & 0.744 \\
\hline isotig26033_1589 & 7 & 37.3 & 19 & 44 & 20 & 0.850 \\
\hline isotig26033_878 & 7 & 37.3 & 19 & 40 & 20 & 0.981 \\
\hline isotig30642_156_Z & 7 & 37.3 & 20 & 42 & 19 & 0.934 \\
\hline isotig28418_576 & 7 & 38.6 & 19 & 43 & 20 & 0.896 \\
\hline isotig29101_440 & 7 & 41.7 & 19 & 43 & 21 & 0.903 \\
\hline isotig29101_970 & 7 & 41.7 & 19 & 43 & 21 & 0.903 \\
\hline isotig30182_1370 & 7 & 41.7 & 19 & 43 & 21 & 0.903 \\
\hline
\end{tabular}


Table 2. Continued.

\begin{tabular}{|c|c|c|c|c|c|c|}
\hline \multirow[b]{2}{*}{ SNP or locus ${ }^{z}$} & \multirow[b]{2}{*}{ Chrom no. } & \multirow[b]{2}{*}{ Position (cM) } & \multicolumn{3}{|c|}{ Observed $^{\mathrm{y}}$} & \multirow[b]{2}{*}{$P$} \\
\hline & & & $\mathrm{a}$ & $\mathrm{h}$ & $\mathrm{b}$ & \\
\hline isotig35377_1073 & 7 & 41.7 & 19 & 43 & 21 & 0.903 \\
\hline isotig26063_757 & 7 & 42.3 & 19 & 42 & 22 & 0.892 \\
\hline isotig28716_2573 & 7 & 49.7 & 22 & 40 & 20 & 0.929 \\
\hline contig00293_452_Z & 7 & 52.2 & 22 & 41 & 20 & 0.947 \\
\hline isotig33385_137 & 7 & 52.8 & 21 & 42 & 20 & 0.982 \\
\hline isotig33385_293_Z & 7 & 52.8 & 21 & 42 & 20 & 0.982 \\
\hline isotig33385_518_Z & 7 & 52.8 & 21 & 42 & 20 & 0.982 \\
\hline isotig36956_785 & 7 & 52.8 & 21 & 36 & 20 & 0.839 \\
\hline isotig25801_1760_Z & 7 & 56.0 & 22 & 41 & 20 & 0.947 \\
\hline isotig28956_1195 & 7 & 56.0 & 22 & 41 & 20 & 0.947 \\
\hline isotig30655_190 & 7 & 56.0 & 22 & 41 & 20 & 0.947 \\
\hline isotig37252_318 & 7 & 63.4 & 21 & 44 & 18 & 0.772 \\
\hline isotig38160_373 & 7 & 71.5 & 24 & 38 & 21 & 0.668 \\
\hline isotig28625_2789 & 7 & 79.6 & 22 & 42 & 19 & 0.892 \\
\hline$G$ locus & 7 & 86.3 & 22 & 46 & 15 & 0.340 \\
\hline isotig37101_618 & 8 & 0.0 & 22 & 40 & 19 & 0.889 \\
\hline isotig27349_313 & 8 & 4.3 & 20 & 40 & 20 & 1.000 \\
\hline isotig34435_676 & 8 & 8.6 & 19 & 45 & 19 & 0.744 \\
\hline isotig34435_689 & 8 & 8.6 & 19 & 42 & 19 & 0.905 \\
\hline isotig30047_888 & 8 & 12.3 & 16 & 43 & 22 & 0.549 \\
\hline isotig30104_1528 & 8 & 12.3 & 16 & 45 & 22 & 0.482 \\
\hline isotig29442_1161_Z & 8 & 17.4 & 14 & 45 & 24 & 0.223 \\
\hline isotig28373_3204 & 8 & 26.7 & 15 & 41 & 27 & 0.175 \\
\hline isotig28373_866 & 8 & 26.7 & 15 & 40 & 27 & 0.169 \\
\hline isotig28412_1084_Z & 8 & 30.5 & 14 & 41 & 28 & 0.094 \\
\hline isotig28412_1177_Z & 8 & 30.5 & 14 & 41 & 28 & 0.094 \\
\hline isotig33362_1094_Z & 8 & 31.7 & 15 & 41 & 27 & 0.175 \\
\hline isotig12913_342 & 8 & 33.5 & 16 & 42 & 25 & 0.375 \\
\hline isotig28432_1209 & 8 & 35.4 & 16 & 41 & 26 & 0.298 \\
\hline isotig28432_1302 & 8 & 35.4 & 16 & 40 & 26 & 0.288 \\
\hline isotig37010_277 & 8 & 35.4 & 16 & 37 & 26 & 0.241 \\
\hline isotig37010_673 & 8 & 35.4 & 16 & 41 & 26 & 0.298 \\
\hline isotig43294_202_Z & 8 & 35.4 & 16 & 41 & 26 & 0.298 \\
\hline isotig37323_396 & 8 & 41.2 & 17 & 36 & 27 & 0.192 \\
\hline isotig20266_1040 & 8 & 53.5 & 15 & 41 & 26 & 0.229 \\
\hline isotig20266_541 & 8 & 53.5 & 15 & 42 & 26 & 0.231 \\
\hline isotig30907_420 & 8 & 55.3 & 15 & 41 & 27 & 0.175 \\
\hline isotig30913_1644_Z & 8 & 55.3 & 15 & 41 & 27 & 0.175 \\
\hline isotig30913_630_Z & 8 & 55.3 & 15 & 41 & 27 & 0.175 \\
\hline isotig30913_822_Z & 8 & 55.3 & 15 & 41 & 27 & 0.175 \\
\hline isotig38873_388 & 8 & 55.3 & 15 & 41 & 27 & 0.175 \\
\hline isotig38873_618 & 8 & 55.3 & 15 & 41 & 27 & 0.175 \\
\hline isotig31862_140_Z & 8 & 56.5 & 15 & 41 & 27 & 0.175 \\
\hline isotig30020_476 & 8 & 59.0 & 15 & 41 & 27 & 0.175 \\
\hline isotig22339_503 & 8 & 60.9 & 17 & 40 & 25 & 0.447 \\
\hline
\end{tabular}

${ }_{\mathrm{z}} \mathrm{Z}$ indicates that the phase of the SNP was unknown and both phases were used for initial mapping, retaining the phase that showed linkage with other phase-known markers. SNPs shown in bold text were mapped for the first time using this segregating family and not previously reported by Duangjit et al. (2013), Damon et al. (2014), or Munaiz and Havey (2020).

${ }^{\mathrm{y}}$ Obs = observed segregations of homozygotes from chartreuse parent (a), homozygotes from B5351 (b), or heterozygotes (h). Codominant segregations at the $G$ locus were determined by scoring bulb colors in $\mathrm{F}_{3}$ families. 


\section{Literature Cited}

Damon, S. and M.J. Havey. 2014. Quantitative trait loci controlling amounts and types of epicuticular waxes in onion. J. Amer. Soc. Hort. Sci. 139:597-602.

Damon, S., R. Groves, and M.J. Havey. 2014. Variation for epicuticular waxes on onion foliage and impacts on numbers of onion thrips. J. Amer. Soc. Hort. Sci. 139:495-501.

Duangjit, J., B. Bohanec, A.P. Chan, C.T. Town, and M.J. Havey. 2013. Transcriptome sequencing to produce SNP-based genetic maps of onion. Theor. Appl. Genet. 126:2093-2101.

Duangjit, J., K. Welsh, M. Wise, B. Bohanec, and M.J. Havey. 2014. Genetic analyses of anthocyanin concentrations and intensity of redbulb color among segregating haploid progenies of onion. Mol. Breed. 34:75-85.

El-Shafie, M. and G. Davis. 1967. Inheritance of bulb color in Allium cepa. Hilgardia 9:607-622.

Green, F.N., R. Baur, M. Thomson, and L. McCarthy. 1997. An example of chartreuse skin colour in onion (Allium cepa L.) cultivar Greenella. Genet. Resources Crop Evol. 44:491-493.

Goldman, I.L., G. Schroeck, and M.J. Havey. 2001. History of public onion breeding programs and pedigree of public onion germplasm releases in the United States. Plant Breed. Rev. 20:67-103.

Havey, M.J. and C. von Kohn. 2017. Efficacy of molecular markers jnurf13 and AcPms1 for prediction of genotypes at the nuclear $M s$ locus in North American open-pollinated populations of onion. HortScience 52:1052-1053.

Havey, M.J. and F. Ghavami. 2018. Informativeness of single nucleotide polymorphisms and relationships among onion populations from important world production regions. J. Amer. Soc. Hort. Sci. 143:34 44.

Jones, H.A. and A. Clarke. 1943. Inheritance of male sterility in the onion and the production of hybrid seed. Proc. Amer. Soc. Hort. Sci. 43:189-194.

Jones, H.A. and L.K. Mann. 1963. Onions and their allies. Interscience, New York, NY.
Khar, A., J. Jakše, and M.J. Havey. 2008. Segregations for onion-bulb colors reveal that red is controlled by at least three loci. J. Amer. Soc. Hort. Sci. 133:42-47.

Kim, S., C.-W. Kim, M. Park, and D. Choi. 2015. Identification of candidate genes associated with fertility restoration of cytoplasmic male sterility in onion (Allium cepa L.) using a combination of bulked segregant analysis and RNA-seq. Theor. Appl. Genet. 128:2289-2299.

Kim, S., M.L. Binzel, S. Park, K.S. Yoo, and L.M. Pike. 2004. Inactivation of DFR (Dihydroflavonol 4-reductase) gene transcription results in blockage of anthocyanin production in yellow onions (Allium cepa). Mol. Breed. 14:253-263.

King, J.J., J.M. Bradeen, O. Bark, J.A. McCallum, and M.J. Havey. 1998. A low-density genetic map of onion reveals a role for tandem duplication in the evolution of an extremely large diploid genome. Theor. Appl. Genet. 96:52-62.

Leksrisompong, P.P., M.E. Whitson, V.D. Truong, and M.A. Drake. 2012. Sensory attributes and consumer acceptance of sweet potato cultivars with varying flesh colors. J. Sens. Stud. 27:59-69.

Masuzaki, S., M. Shigyo, and N. Yamauchi. 2006. Complete assignment of structural genes involved in flavonoid biosynthesis influencing bulb color to individual chromosomes of shallot (Allium cepa L.). Genes Genet. Syst. 81:255-263.

Munaiz, E.D. and M.J. Havey. 2020. Genetic analyses of epicuticular waxes associated with the glossy foliage of 'White Persian' onion. J. Amer. Soc. Hort. Sci. 145:67-72.

van Ooijen, J.W. 2006. JoinMap 4, software for the calculation of genetic linkage maps in experimental populations. Kyazma, Wageningen, the Netherlands.

von Kohn, C., A. Kiełkowska, and M.J. Havey. 2013. Sequencing and annotation of the chloroplast DNAs of normal $(\mathrm{N})$ male-fertile and male-sterile (S) cytoplasms of onion and single nucleotide polymorphisms distinguishing these cytoplasms. Genome 56:737-742. 